only a short course, and leave no local deformity of the chest wall. With effusion the pyrexia may continue 10,14 , or even 28 days, and the fluid is either absorbed, or, on removal by paracentesis; does not as a rule re-accumulate. The deformity left even by a large effusion is not great. In children no defect may be appreciable after a few weeks' convalescence, and even in adults whilst the chest movements and the breath sounds may be deficient for a time, there is no marked or permanent deformity. The fluid in non-tuberculous pleurisy as a rule coagulates spontaneously, and gives evidence of the presence of the pneumococcus, staphylococcus, or streptococcus. In tuberculous pleurisy, on the other hand, the acute stage is longer, recovery is slower, and there is a decided tendency to marked deformity of the chest. In the slighter cases there is pyrexia, showing an evening rise and morning fall, with friction sound, the latter usually in the lower part of the axilla. As the febrile process continues, defective movement, dull percussion, weak respiratory murmur, and retraction of the chest wall are noted, and the friction sound disappears. These local signs are due to thickening of the pleura, and puncture of the chest shows the absence of either serous or purulent effusion. Such cases may recover, or a subacute general tuberculosis or chronic disease of the lung may develop. When tuberculous pleurisy is associated with effusion the diagnosis from the non-tuberculous variety may be very difficult. The following considerations are of value:(1) In tuberculous cases pyrexia may continue for weeks and months, and without the development of empyema; (2) A second, third, or even further paracentesis is usually required; (3) the fluid, when removed, sometimes coagulates spontaneously, but in long-continued cases coagulation is slight or absent. The fluid may sometimes contain the bacteria which are the cause of non-tuberculous, primary pleurisy ; but if these are absent, that is, if the fluid is sterile by cultivation on ordinary media, the disease is tuberculous; or the presence of tubercle bacilli may be proved by microscopical examination or inoculation of the deposit from the centrifugalised fluid. In some few cases the tuberculous nature of a pleurisy is shown by extension of the disease to the peritoneum, as shown by the gradual appearance of tumidity of the abdomen, or by the presence of some fluid effusion. Much more common is extension to the lung or co-incident tuberculous disease of the lung as shown by the detection of tubercle bacilli in the sputa. But all cases of pleural effusion with expectoration are not tuberculous. Pneumococcal and streptococcal pleurisies may have this symptom, but here again the examination of the sputa reveals the diagnosis. As persistent effects of a tuberculous pleurisy may be seen considerable retraction of the chest wall, diminished entrance of air, defective resonance (modified, perhaps, by local compensatory emphysema), and signs of dilatation of the bronchi. These signs may persist even though the patient's general condition is that of physical well-being, and there is no expectoration.

In reference to treatment, rest in bed and good nursing are, of course, indicated. The important point is the question of paracentesis, and it may be said that there is no more danger in drawing off the fluid in tuberculous than in non-tuberculous cases. Thus, apart from considerations of urgency, as, for example, the existence of respiratory distress, it is good practice to draw off the fluid when it is large in quantity and shows no tendency to spontaneous absorption. With prolonged pyrexia and the absence of urgent symptoms the patient should rest in the open air, being at the same time well wrapped up and well fed, and open-air treatment after une pyrexia has ceased is the best method to promote healing of the tuberculous lesion and to prevent recurrences.

Lancet, March 3, 1906.

\section{THE TREATMENT OF CHRONIC RENAL} DISEASE.

IN a paper on this subject read before the Medical Society of London Dr. Samuel West considered principally points on which modern opinion is undecided or conflicting. Dealing first with chronic parenchymatous nephritis, the writer said the problem was how to promote the growth of the renal cells. To secure this end effective nutrition was necessary, and this was impossible if a too prolonged course of purely milk diet was practised. Meat extracts and alcohol should always be excluded, but this restriction did not apply to eggs in moderation, and there was no sufficient reason why white meat should be ordered and red meat always forbidden. Similarly, confinement to bed or to one room was often too long persisted in. Under a more liberal diet and open-air treatment (short of exposure to chill) rapid improvement often took place. In granular kidney the treatment must differ in each of the three stages of the malady. In the earliest stage much might be done to retard the progress of the disease. Over-exertion and exposure should be avoided, but moderate, regular exercise was desirable. In the middle stage of cardiac hypertrophy and thickened arteries treatment should be directed to prevent the risks of cardiac failure, rupture of vessels, and intercurrent nephritis. Hence the necessity of avoiding strain, whether physical or mental. If arterial tension became subnormal, digitalis might be necessary, but otherwise, though useful for the heart, it increased peripheral resistance by contracting the arterioles. On the other hand caffeine acted as a cardiac stimulant without contracting the vessels. In the later stages of the disease the symptoms became, more pronounced, and might arise in almost any part of the body. Arterial tension now, varied greatly. If low, it must be raised by diet, stimulants, digitalis, ergot, and adrenalin. High tension, on the other hand, demanded modification of the diet in the opposite direction; also purgation, baths, diaphoretics, nitro-glycerine, and potassium iodide. Pilocarpine is a most useful drug, and its use is free from the risks sometimes said to attend it;... it is particularly valuable for restlessness, sleeplessness, and threatening uræmia. As a hypnotic cannabis indica is of service. In some cases the administration of renal extract seemed to do good.

In the discussion which followed Dr. West's 
paper Sir Lauder Brunton advocated the use of strophanthus in the middle stage of the disease as this drug acted as a cardiac tonic without constricting the blood-vessels. With high arterial tension 20 grains of potassium nitrate and 1 grain of sodium nitrite in a tumblerful of water every morning was useful; it lowered the tension, and might without harm be continued for years. Dr. Haviland Hall considered that milk diet should always have a fair trial-three to four weeks-in chronic nephritis. He also emphasised the value of pilocarpine. Ir granular kidney he used strophanthus in combination with potassium iodide. Headache associated with high tension would frequently be relieved by one-third of a minim of solution of trinitrin, frequently repeated if necessary.

\section{Progress in Medicine and SURGery.}

\section{DISEASES OF THE NERVOUS SYSTEM.}

(Continued from page 353.)

Cerebral Arteriosclerosis.-Notwithstanding the facts that arteriosclerosis of the cerebral vessels is not uncommon in various forms of mental disease, especially in later life, and that on the other hand it may undoubtedly be present without causing any recognisable disease of the mind, A. M. Barrett ${ }^{5}$ thinks there is sufficient ground for establishing as a distinct entity an arteriosclerotic dementia. This group shows clinically a prominence of symptoms referable to focal or diffuse vascular brain disease which are found anatomically to be associated with destructive lesions of the brain due to diseased vessels. In those familiar cases of dementia due to such coarse lesions as hæmorrhage and softening, and described as post-paralytic, post-apoplectic, and arteriosclerotic, it will usually be found that an arteriosclerotic condition of the finer as well as of the larger vessels is present, together with more or less destruction of the cortical nervous elements. Alzheimer in 1902 described four groups of cases in which the mental process is associated with arteriosclerotic brain disease, all, however, as Barrett points out, being varieties of one process. Group I. consists in arteriosclerotic brain atrophy, with symptoms either mild or severe. In the milder cases pronounced increase of mental fatigue, memory weakness, headache, and dizzy attacks are observed, and on examination severe sclerosis of the arteries is revealed with some overgrowth of neuroglial tissue, but no gross changes. In the more severe form the symptoms are graver and progress to deep dementia, and epileptic convulsions or apoplectic attacks occur. Group II. consists of subcortical encephalitis, an atrophy of the deep-lying white substance due to sclerosis of the long medullary arteries. It has been specially described by Binzwanger. Interference with the association processes is the first and most striking symptom. Speech is affected early, and there are various aphasic disturbances with mental confusion and convulsive attacks. In Group III. there is a perivascular gliosis and consequent atrophy of nervous elements in areas of which the nutrition is impaired by reason of the sclerosis of the arteries supplying the parts. In Group IV. senile brain atrophy is present, showing as a disintegration of the nervous elements in peculiar wedgeshaped foci, or in streaks, and due to an arteriosclerotic degeneration of the smaller vessels of the cortex. In the production of these types, which are usually somewhat obscured by the occurrence of focal lesions, two processes must be considered-the changes in the vessels and the reactions in the nervous tissue. In the larger vessels of the brain the changes are similar to those found in arteriosclerotic vessels elsewhere. The intima is chiefly affected, becoming irregularly thickened by proliferation of cells and elastic tissue by fibrous new formation. Later, necrotic degenerative processes lead to atheromatous degeneration, or more rarely hyaline transformation. In the finer vessels the endothelial cells proliferate and the elastic fibres multiply or split, leading sometimes to occlusion of the original lumen of the vessel and the formation in it of new channels; as many as four new channels have been observed running through the proliferated endothelium. The cross section of such vessels gives the appearance of clusters or chains of rings. In the nerve tissue, as a result of these changes, focal softenings occur from the sudden shutting off of nutrition, or in cases of more gradual deprivation partial softening and degeneration of the less resistant nervous elements with overgrowth of the glia. The hyaline degeneration leads to fine miliary aneurysms, which by rupture produce the larger cerebral hæmorrhages. Epithelioid cells are usually present in arteriosclerotic foci. Arteriosclerosis may be present as a purely associated condition in general paralysis, and in senile atrophy. From the former it is to be histologically differentiated by the less diffuse character of the process, and above all by the absence of lymphoid and plasma cell infiltration of the vessel wall. In senile atrophy, which may occur without arteriosclerosis, the disappearance of the nerve cells is also more diffuse and general, while in the dementia due to vascular change the cortex shows a patch-like degeneration, and the lesions are focal in character and related to the distribution of the arteries. In senile arteriosclerotic dementia there are observable, clinically, among other symptoms, forgetfulness, loss of judgment, delusions of persecution, and apathy deepening into a profound dementia. These symptoms are common in non-vascular senile dementia, but in addition, where the vessels are at fault, are observed "shocks," focal paralysis, speech defects, articulatory and aphasic, and the constitutional signs of arteriosclerosis. Characteristics of the disease as described in Group I. are weakness of the retentive memory-an early symptom-and marked dullness and lack of productivity, rapid alternation of moods, but no constant depression; all moods tending decidedly to marked apathy and indifference to surroundings. In many respects these cases very 